\title{
Febuxostat for the Prevention of Recurrent 2,8-dihydroxyadenine Nephropathy due to Adenine Phosphoribosyltransferase Deficiency Following Kidney Transplantation
}

\author{
Koji Nanmoku, Akira Kurosawa, Takahiro Shinzato, Toshihiro Shimizu, \\ Takaaki Kimura and Takashi Yagisawa
}

\begin{abstract}
Adenine phosphoribosyltransferase (APRT) deficiency is a rare autosomal recessive disorder that results in irreversible renal damage due to 2,8-dihydroxyadenine (DHA) nephropathy. A 28-year-old man underwent living-related kidney transplantation for chronic kidney disease of unknown etiology. Numerous spherical brownish crystals observed in his urinary sediment on postoperative day 3 and were observed within the tubular lumen of renal allograft biopsy specimens on postoperative day 7. After a genetic diagnosis, febuxostat treatment was started on postoperative day 7 , with the dosage gradually increased to $80 \mathrm{mg} /$ day until complete the disappearance of 2,8-DHA crystals. Febuxostat prevented secondary 2,8-DHA nephropathy after kidney transplantation.
\end{abstract}

Key words: adenine phosphoribosyltransferase deficiency, 2,8-dihydroxyadenine nephropathy, nephrolithiasis, xanthine dehydrogenase inhibitor, febuxostat, kidney transplantation

(Intern Med 56: 1387-1391, 2017)

(DOI: 10.2169/internalmedicine.56.8142)

\section{Introduction}

Adenine phosphoribosyltransferase (APRT) is an enzyme involved in purine metabolism: it catalyzes the formation of adenosine monophosphate and inorganic pyrophosphate from adenine and 5-phosphoribosyl-1-pyrophosphate (1). Because APRT represents the only pathway for adenine metabolism, the absence of APRT activity results in the accumulation of adenine, which is instead catabolized by xanthine dehydrogenase $(\mathrm{XDH})$ to 8-hydroxyadenine and then to 2,8-dihydroxyadenine (DHA), leading to nephrolithiasis and crystalline nephropathy (2-4).

The APRT gene, which is located on chromosome 16q24, is approximately $2.6 \mathrm{~kb}$ long, contains 5 exons and 4 introns, and encodes the 180-amino acid protein APRT (5). The mutant alleles responsible for type I and II APRT deficiency are classified as APRT*Q0 (complete enzyme deficiency in vivo and in vitro) (1) and APRT*J (complete en- zyme deficiency in vivo but partial deficiency in vitro), respectively. Type II APRT deficiency is mainly observed in Japanese patients (6).

APRT deficiency is a rare (at least 1:50,000 to $1: 100,000$ ) autosomal recessive disorder that results in irreversible renal damage and subsequent chronic kidney disease (CKD) due to the deposition of 2,8-DHA in the renal tubules (5). Unfortunately, APRT deficiency is under-recognized by physicians, and its diagnosis is often delayed for many years. In patients with CKD, APRT deficiency is frequently misdiagnosed as non-genetic kidney stones, chronic interstitial nephritis, obstructive nephropathy, or hypertensive nephrosclerosis (3). A timely diagnosis of APRT deficiency is crucial, since early treatment with the allopurinol (an XDH inhibitor) can effectively prevent further nephrolithiasis and stabilize the renal function $(2,4)$.

This is the first report describing the use of a recently introduced nonpurine XDH inhibitor, febuxostat, in a patient who developed 2,8-DHA crystals soon after kidney trans- 

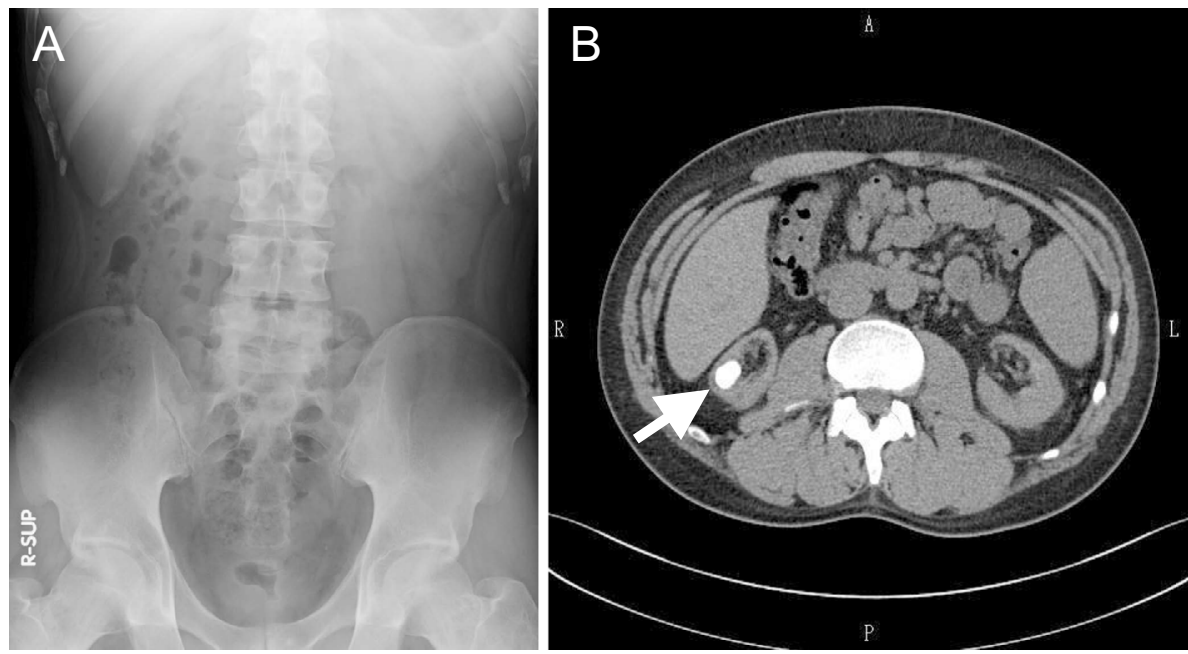

Figure 1. Pre-transplant examinations of the abdomen by X-ray and computed tomography. A small area of calcification (arrow) in the lower pole of the right kidney not observed on the X-ray film (A) but was detected on computed tomography (B).
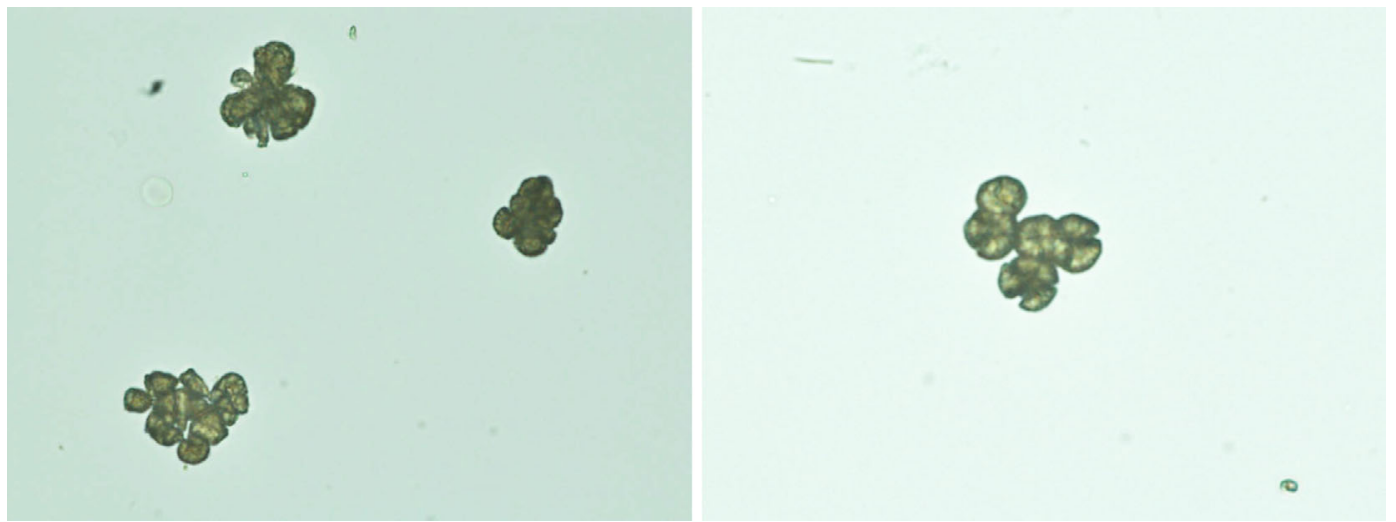

Figure 2. Numerous spherical brownish crystals in the urinary sediment on postoperative day 3 (magnification $\times \mathbf{4 0 0}$ ).

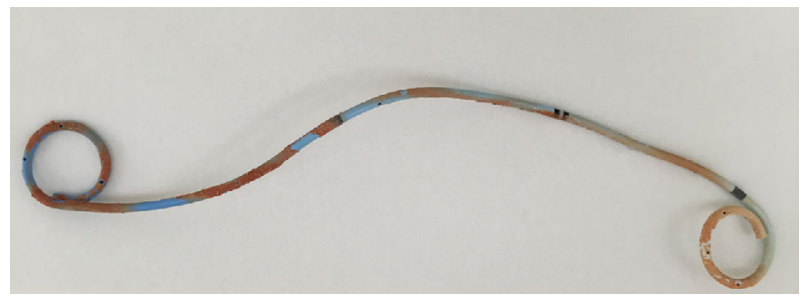

Figure 3. Brown debris adhering to the double-J ureteral stent was observed on postoperative day 7 .

plantation. We found that febuxostat prevented the recurrence of 2,8-DHA crystals and nephrolithiasis due to APRT deficiency.

\section{Case Report}

The patient was a 28-year-old Japanese man who underwent 5 years of hemodialysis for CKD of unknown etiology. Despite a history that included several episodes of urinary stone excretion during childhood, a detailed examination had not been previously performed. The deterioration of the patient's renal function was not identified until he was diagnosed with stage $5 \mathrm{CKD}$; thus, a renal biopsy had never been considered. There was no family history of kidney disease. Only a small area of calcification in the lower pole of the right kidney was observed by computed tomography at the time of the pre-transplant examination (Fig. 1). With the exception of mild hypospadias, there were no abnormal findings. The patient underwent living-related kidney transplantation from his mother. The combination of tacrolimus extended-release, mycophenolate mofetil, and methylprednisolone was administered as maintenance immunosuppressive therapy after induction therapy with basiliximab. A double-J ureteral stent was inserted at the time of ureteroneocystostomy due to atrophic bladder. The renal graft started to function immediately, and a sufficient urine volume was obtained; however, the urine was already cloudy on postoperative day 3, and the analysis of the urinary sediment showed numerous spherical brownish crystals (Fig. 2). The brown debris adhering to the double-J ureteral stent was removed on postoperative day 7 (Fig. 3) and was identified as 

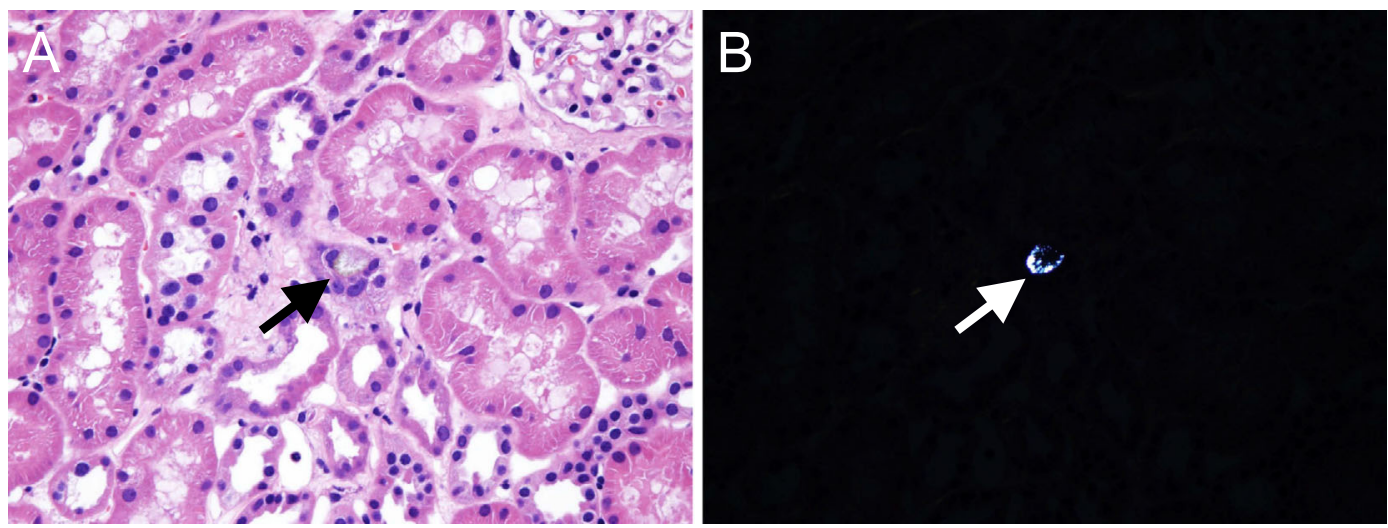

Figure 4. The findings of renal allograft biopsy obtained on postoperative day 7. Small crystal deposits within the tubular lumen of the renal allograft biopsy segment by conventional light microscopy (A) and polarized light microscopy (B) (magnification $\times \mathbf{4 0 0}$ for both). Polarized light microscopy clearly shows the crystals. Crystals deposited within the tubular lumen of the renal allograft biopsy specimen had completely disappeared by postoperative day 201.

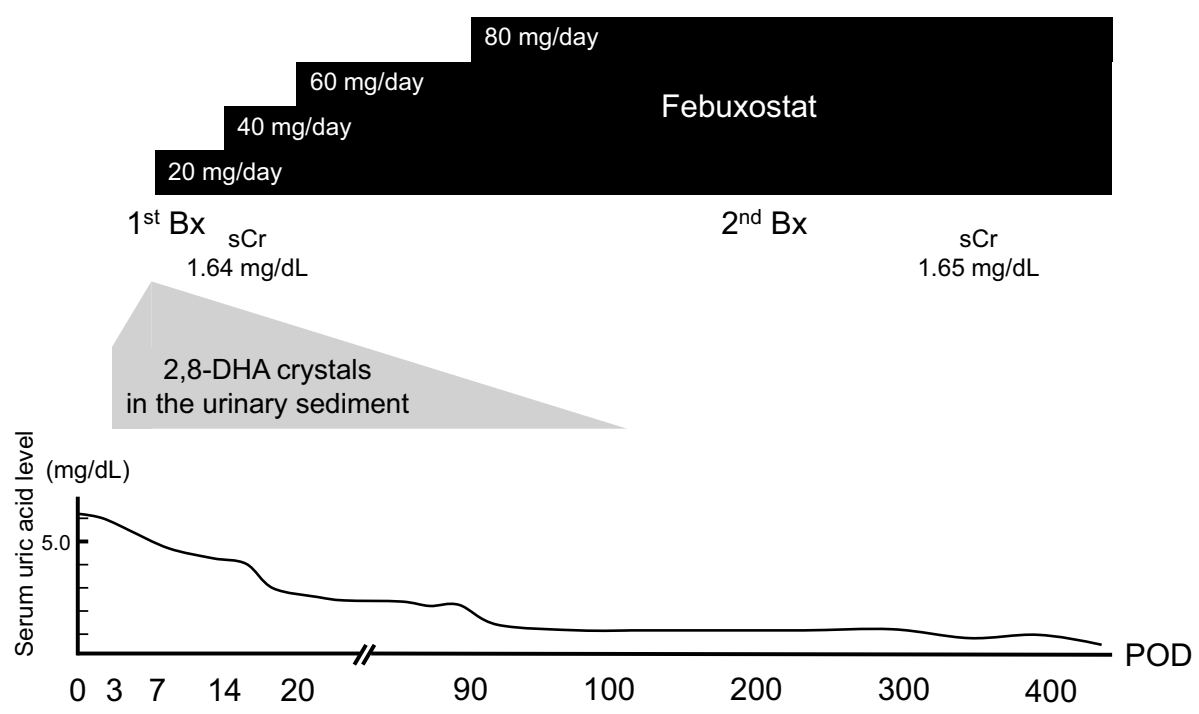

Figure 5. The clinical course after kidney transplantation. The dose of febuxostat was gradually increased until the disappearance of 2,8-DHA crystals from the urinary sediment. Maintenance therapy with febuxostat $(80 \mathrm{mg} / \mathrm{day})$ was associated with a stable renal allograft function following the disappearance of 2,8-DHA crystals from the urinary sediment and renal allograft biopsy specimens.

2,8-DHA by infrared spectroscopy using the $\mathrm{KBr}$ wafer technique. Similar small crystal depositions were observed within the tubular lumen in a renal allograft biopsy specimen obtained on postoperative day 7 (Fig. 4). The complete absence of APRT enzymatic activity was suspected based on the peripheral blood mononuclear cells that were isolated from the patient and which were cultured in the presence of phytohemagglutinin, interleukin 2, and 6methylpurine $(7,8)$. A mutational analysis of the $A P R T$ gene was performed after receiving written informed consent from the patient, and the genetic diagnosis of $\mathrm{APRT}^{*} \mathrm{Q} 0 /$ APRT*Q0 (a nonsense mutation from TGG to TGA at codon 98) was confirmed by a polymerase chain reactionrestriction fragment length polymorphism analysis and DNA sequencing (9).
Febuxostat (initial dose: $20 \mathrm{mg} /$ day) was started 7 days after kidney transplantation to remove the 2,8-DHA crystals (Fig. 5). In addition, the patient was encouraged to hydrate and was put on a purine-restricted diet. He was discharged from our hospital on postoperative day 14. At that time, his serum creatinine level was $1.64 \mathrm{mg} / \mathrm{dL}$ with a febuxostat dose of $40 \mathrm{mg} /$ day. The dosage of febuxostat was gradually increased to achieve the complete removal of the 2,8-DHA crystals from his urine. At 110 days after kidney transplantation, the dose of febuxostat was $80 \mathrm{mg} /$ day and there was no evidence of 2,8-DHA crystals in the urinary sediment specimen. Accordingly, the dose of febuxostat was maintained at $80 \mathrm{mg} /$ day. No febuxostat-related side effects (such as liver dysfunction) were observed during follow-up. The second renal allograft biopsy and urinalysis on postoperative 
day 201 demonstrated the absence of 2,8-DHA crystals. The renal allograft function remained stable with the dose of febuxostat, maintained at $80 \mathrm{mg} /$ day. The patient's serum creatinine level at the end of the first postoperative year was $1.65 \mathrm{mg} / \mathrm{dL}$. The patient's serum uric acid levels were 6.2 $\mathrm{mg} / \mathrm{dL}$ before kidney transplantation, $5.0 \mathrm{mg} / \mathrm{dL}$ at postoperative day 7 before the administration of febuxostat, and $1.3 \mathrm{mg} / \mathrm{dL}$ with febuxostat (80 mg/day) (Fig. 5).

\section{Discussion}

The clinical course of the present case highlights two critical clinical issues. First, APRT deficiency is an important etiology of CKD as 2,8-DHA nephrolithiasis recurs immediately after kidney transplantation. Secondly, it underscores the more urgent issue in the underappreciation of APRT deficiency by clinicians (10). Thus, patients with a past history of urolithiasis during childhood should be evaluated for potential APRT deficiency.

Primary hyperoxaluria is an important cause of pediatric urolithiasis associated with CKD. Since both APRT deficiency and primary hyperoxaluria are autosomal recessive, the family history is often negative (3). Marked renal calcification, which is detectable by X-ray in patients with primary hyperoxaluria, is absent in patients with APRT deficiency. Thus, immediate genetic testing and a urinalysis should be conducted for the differential diagnosis when patients have a history of urolithiasis and CKD. Genetic testing plays an important role in the definitive diagnosis of most patients with anuria and urolithiasis during childhood. Ideally, a genetic diagnosis of APRT deficiency should be made before kidney transplantation, and XDH inhibitor treatment should be initiated immediately after kidney transplantation to prevent the recurrence of 2,8-DHA nephrolithiasis. In the present case, it was not possible to diagnose the patient's APRT deficiency prior to kidney transplantation. Although it is not commonly observed in association with APRT deficiency, the appearance of spherical brownish crystals in the urinary sediment on postoperative day 3 early after transplantation was instrumental in raising the suspicion of APRT deficiency.

The recently introduced nonpurine $\mathrm{XDH}$ inhibitor febuxostat can effectively prevent the recurrence of 2,8-DHA nephrolithiasis after kidney transplantation. Thus far, another $\mathrm{XDH}$ inhibitor, allopurinol, has been used to prevent both primary (4) and secondary (2) 2,8-DHA nephrolithiasis following kidney transplantation. It is not always possible to achieve an effective dose of allopurinol, especially in patients with CKD, due to the increased risk of allopurinol hypersensitivity syndrome (4). In kidney transplant recipients, an insufficient dose of allopurinol (100-200 mg/day) was associated with graft loss due to recurrent 2,8-DHA nephrolithiasis (2). Previously, febuxostat was administered after allopurinol to APRT-deficient patients with allopurinol hypersensitivity syndrome (4) and to patients in the maintenance period after kidney transplantation (2) as a second- line therapy. This is the first report of the administration of febuxostat immediately after kidney transplantation in order to remove 2,8-DHA crystals and to prevent their recurrence. The dose of febuxostat was safely increased to $80 \mathrm{mg} /$ day until the complete disappearance of 2,8-DHA crystals, which was determined by a urinalysis and biopsy of the renal allograft. A crystalluria study by light and polarizing microscopy represents a noninvasive, rapid, cheap and efficient diagnostic test (3). Because there is no specific examination, such as a biochemical test to qualify the 2,8-DHA crystals, we continued to observe the patient to confirm that there was no further recurrence of 2,8-DHA crystals in the urinary sediment. Accordingly, febuxostat proved safer and more efficient than allopurinol in preventing recurrent 2,8-DHA nephrolithiasis after kidney transplantation. Along with XDH inhibiting therapy, an increased fluid intake and a low purine diet are usually recommended; however, the extent to which these decrease the excretion of DHA is not known (3). Since DHA, unlike uric acid, remains very insoluble at high $\mathrm{pH}$ values, urine alkalinization is not recommended (3).

In conclusion, APRT deficiency should be considered in kidney transplant candidates with uncertain CKD etiology. Clinically effective doses of febuxostat should be administered as soon as possible following the diagnosis of APRT deficiency in order to prevent secondary 2,8-DHA nephropathy following kidney transplantation.

\section{The authors state that they have no Conflict of Interest (COI).}

\section{Acknowledgement}

The authors would like to thank Dr. Atsuo Taniguchi and Ms. Chieko Sekita from the Institute of Rheumatology, Tokyo Women's Medical University for the diagnosis of APRT deficiency by cell and mutational analyses.

\section{References}

1. Edvardsson V, Palsson R, Olafsson I, Hjaltadottir G, Laxdal T. Clinical features and genotype of adenine phosphoribosyltransferase deficiency in Iceland. Am J Kidney Dis 38: 473-480, 2001.

2. Zaidan M, Palsson R, Merieau E, et al. Recurrent 2,8dihydroxyadenine nephropathy: a rare but preventable cause of renal allograft failure. Am J Transplant 14: 2623-2632, 2014.

3. Bollée G, Cochat P, Daudon M. Recurrence of crystalline nephropathy after kidney transplantation in APRT deficiency and primary hyperoxaluria. Can J Kidney Health Dis 2: 31, 2015.

4. Runolfsdottir HL, Palsson R, Agustsdottir IM, Indridason OS, Edvardsson VO. Kidney disease in adenine phosphoribosyltransferase deficiency. Am J Kidney Dis 67: 431-438, 2016.

5. Valaperta R, Rizzo V, Lombardi F, et al. Adenine phosphoribosyltransferase (APRT) deficiency: identification of a novel nonsense mutation. BMC Nephrol 15: 102, 2014.

6. Kamatani N, Terai C, Kuroshima S, Nishioka K, Mikanagi K. Genetic and clinical studies on 19 families with adenine phosphoribosyltransferase deficiencies. Hum Genet 75: 163-168, 1987.

7. Kamatani N, Takeuchi F, Nishida Y, et al. Severe impairment in adenine metabolism with a partial deficiency of adenine phosphoribosyltransferase. Metabolism 34: 164-168, 1985.

8. Hakoda M, Yamanaka H, Kamatani N, Kamatani N. Diagnosis of 
heterozygous states for adenine phosphoribosyltransferase deficiency based on detection of in vivo somatic mutants in blood $\mathrm{T}$ cells: application to screening of heterozygotes. Am J Hum Genet 48: 552-562, 1991.

9. Kuno S, Taniguchi A, Saito A, Tsuchida-Otsuka S, Kamatani N Comparison between various strategies for the disease-gene mapping using linkage disequilibrium analyses: studies on adenine phosphoribosyltransferase deficiency used as an example. J Hum Genet 49: 463-473, 2004.
10. Nasr SH, Sethi S, Cornell LD, et al. Crystalline nephropathy due to 2,8-dihydroxyadeninuria: an under-recognized cause of irreversible renal failure. Nephrol Dial Transplant 25: 1909-1915, 2010.

The Internal Medicine is an Open Access article distributed under the Creative Commons Attribution-NonCommercial-NoDerivatives 4.0 International License. To view the details of this license, please visit (https://creativecommons.org/licenses/ by-nc-nd/4.0/).

(C) 2017 The Japanese Society of Internal Medicine http://www.naika.or.jp/imonline/index.html 